\title{
fMRI Feature Extraction Model for ADHD Classification Using Convolutional Neural Network
}

\author{
Senuri De Silva, University of Moratuwa, Sri Lanka \\ Sanuwani Udara Dayarathna, University of Moratuwa, Sri Lanka \\ Gangani Ariyarathne, University of Moratuwa, Sri Lanka \\ Dulani Meedeniya, University of Moratuwa, Sri Lanka \\ iD https://orcid.org/0000-0002-4520-3819 \\ Sampath Jayarathna, Old Dominion University, USA
}

\begin{abstract}
Biomedical intelligence provides a predictive mechanism for the automatic diagnosis of diseases and disorders. With the advancements of computational biology, neuroimaging techniques have been used extensively in clinical data analysis. Attention deficit hyperactivity disorder (ADHD) is a psychiatric disorder, with the symptomology of inattention, impulsivity, and hyperactivity, in which early diagnosis is crucial to prevent unwelcome outcomes. This study addresses ADHD identification using functional magnetic resonance imaging (fMRI) data for the resting state brain by evaluating multiple feature extraction methods. The features of seed-based correlation (SBC), fractional amplitude of low-frequency fluctuation (fALFF), and regional homogeneity ( $\mathrm{ReHo}$ ) are comparatively applied to obtain the specificity and sensitivity. This helps to determine the best features for ADHD classification using convolutional neural networks (CNN). The methodology using fALFF and ReHo resulted in an accuracy of $67 \%$, while SBC gained an accuracy between $84 \%$ and $86 \%$ and sensitivity between $65 \%$ and $75 \%$.
\end{abstract}

\section{KEYWORDS}

ADHD, Applied Computing, Bio-Medical Science, Computational Biology, Knowledge Engineering, Neuroimaging, Seed-Based Correlation Classification Model

\section{INTRODUCTION}

At present, biomedical intelligence for clinical data analysis is readily used for health informatics research and applications. With the technology advancements, the novel computational methods support to process large amounts of health-care data. Biomedical imaging is used to derive features and identify patterns using various analysis techniques on clinical data for medical purposes. Thus, the diagnosis process of medical untidiness can be supported by an appropriate selection and processing of image features. Recently, the use of biomedical imaging for the classification of psychiatric disorders has been considered for a precise diagnosis process.

Attention deficit hyperactivity disorder (ADHD) is a prevalent neurological disorder among children. There is a prospect of carrying the associated behavioural, communication and learning 
issues of such children into adulthood and comorbid with other neurological disorders (Meedeniya \& Rubasinghe, 2020). ADHD can be described as the tenacity pattern of inattention, hyperactive or impulsivity that is notably higher than the corresponding development groups, as defined by the DSM-5 diagnostic criteria (American Psychiatric Association, 2013). At present, ADHD covers 5\% of the entire child population causing impairments of their childhood, and $70 \%$ carries them into adulthood and a higher rate of comorbid with other neurological disorders (Polanczyk, de Lima, Horta, Biederman \& Rohde, 2007). This is the main motivation of this study to propose a method for the early identification of ADHD using medical imaging. Generally, psychiatric disorders such as depression, anxiety, learning disorder, obsessive-compulsive disorder, conduct disorder, and other learning-related difficulties are some of the highly comorbid disorders with ADHD (De Silva, Dayarathna, Ariyarathne, Meedeniya \& Jayarathna, 2019a; Meedeniya \& Rubasinghe, 2020).

Among psychophysiological measures, electroencephalography (EEG) has shown the possibility of identifying the ADHD subtypes; inattentively, impulsivity and hyperactivity efficiently (Kaur, Arun, Singh \& Kaur, 2018). However, the source reconstruction is not a well-posed problem and contain inherent limitations on spatial resolution. Thus, using EEG is not alone applicable in the deification process for a larger scale and combining heterogeneous sources (Abreu, Leal \& Figueiredo, 2018). On the other hand, fMRI tests have been used widely providing effective identification process, and effectivity features than EEG by capturing the brain response in the presence of neural stimuli. Therefore, this study addresses the feature extraction process of fMRI data for ADHD classification.

fMRI data are used to identify the abnormal neural activities of ADHD subjects in the resting state of the selected brain regions (Metin et al., 2015). Hence, this paper focuses on the ADHD identification using fMRI data based on features such as fractional Amplitude of Low-Frequency Fluctuation (fALFF), Regional Homogeneity (ReHo) and seed-based correlation (SBC) to extract the regional activities in the brain. The proposed solution addresses Default Mode Networks (DMN) based brain regions to study the abnormal activities and extract features using the SBC technique as the main contribution. This study evaluates various features from fMRI image data by comparing the use of preprocessed and un-processed data in the classification process. The preprocessing of raw fMRI data and extraction of optimal features also contribute to the novelty of this study. The approach uses CNN as the learning model to derive an accurate model with fMRI data. This is developed as a prototype named ADHD-Care_v2 (ADHD-care, 2019). This is an extension of our previous study of ADHD diagnosis with eye movement data using a rule-based system (De Silva et al., 2019b).

The paper is structured as follows. Section 2 explains the theoretical approaches applied in the proposed solution along with the practical application of ADHD classification. Section 3 presents the design of the solution including the system workflow. Section 4 includes the explanation of the methodology supported by the comprehensive implementation. Then Section 5 comparatively evaluates the results and Section 6 concludes the paper.

\section{THEORETICAL BACKGROUND}

\section{Attention Deficit Hyperactive Disorder}

ADHD is a common psychiatric disorder among children. Children with ADHD also tend to exhibit a variety of symptoms with different severity levels and behaviours. The most common symptoms are issues in attention, hyperactivity, impulsivity, including failures in inhibition monitoring of the brain. This may also cause to lessen the self-control and confidence of children as there is a high probability of continuing the disorder to their adulthood by leading them into other severe comorbidity disorders (De Silva et al., 2019a; Meedeniya \& Rubasinghe, 2020; Polanczyk et.al, 2007). Thus, early detection of ADHD is important to reduce further impairments and negative impact on their day to day life.

The identification process of ADHD is still using subjective measures where manual clinical approaches are applied. Hence well-defined and comprehensive objective approaches are being 
motivated in the research to achieve high accuracy, specifically a high sensitivity in their resulting models of identification. Several psychophysiological metrics such as eye movement data, electroencephalography (EEG), Electromyography (EMG), Magnetic Ray Imaging (MRI), and Functional Magnetic Ray Imaging (fMRI) have been used in ADHD identification process (De Silva et al., 2019b; Kaur et al., 2018; Siuly \& Zhang, 2016).

\section{Neuroimaging Metrics: fMRI}

Functional Magnetic Resonance Imaging (fMRI) helps to measure the changes in blood oxygen levels result from the neural activities in the brain (Glover, 2011). Brain's functional anatomy can be examined using fMRI to evaluate the diseases by detecting abnormalities in the brain. Magnetic Resonance Imaging (MRI) uses radiofrequency pulses and a powerful magnetic field to capture all the internal body structures. Generally, MRI can determine certain diseases by evaluating different parts of the body such as bone damage and tumour images. The fMRI uses MRI to measure the sensitive changes in blood flow which is in the active part of the brain. fMRI data (Mao et al., 2019) has emphasized the capability of identifying various patterns of mental functionalities of brain activation. Several studies have analyzed fMRI data to detect the correlation between the task which is performed by the subject during the scan and the brain activation (Meedeniya \& Rubasinghe, 2020; Rubasinghe \& Meedeniya, 2020; Mao et al., 2019; Peng, Lin, Zhang \& Wang, 2013).

\section{Data Preprocessing Applications}

SPM12 (Statistical Parametric Mapping) is an open-source free package that runs on the MATLAB environment (Ashburner et al., 2014). SPM12 uses the NIFTI format 3D images. SPM12 mainly supports spatial preprocessing function, statistical functions, and visualization. This is a well-developed tool with accurate and robust results. The user requires a basic prior knowledge only and the tool is easy to learn. The prime functions of SPM12 are realigning, slice timing correlation, coregistration, normalizing and smoothing. It also provides different options to separately perform processing in each step and provides the freedom to the user to change the default values of the steps, through attribute selection. Thus, processing tasks can perform seamlessly. One notable feature in this tool is that it supports batch processing, which allows applying a batch of fMRI images into the pipeline and retrieves results. However, this tool requires MATLAB distribution to achieve optimal results.

The Configurable Pipeline for the Analysis of Connectomes (C-PAC) is an open-source, configurable automated processing pipeline for fMRI where users can reliably preprocess and analyze data (Craddock et al., 2013). C-PAC has been commonly used for large datasets as it supports a variety of preprocessing strategies for different subjects. These functionalities include realignment, slicetime correction, normalization, coregistration, smoothing, motion scrubbing and skull stripping. The Nipype pipelining library of python has been used to implement the C-PAC. It contains advanced analysis methods for the comprehensive exploration of the network structure, connectivity patterns and brain-behaviour relations. C-PAC contains the individual-level measures of ALFF, fALFF, SBC analysis, network centrality and voxel-mirrored homotopic connectivity. In performance-wise, C-PAC supports shared memory and cluster-based computing environments, which have high performance to reduce the computation time. However, the tool requires expert knowledge and the configuration is complex.

\section{Seed Based Correlation}

In the study of brain dynamics, measuring the connectivity is essential in generating the connectivity maps. The connectivity maps carry the features to the model creation later. The seed-based correlation (SBC) method particularly incorporates defining the functional connectivity of the brain. The functional connectivity is further known as the temporal activity in brain dynamics. Moreover, blood-oxygen-level-dependent imaging (BOLD), is a method used in fMRI to identify the active brain regions. As fMRI is generated by BOLD signals, the time series can be generated for those signals. 
A selected seed area of interest (ROI) can be used to calculate the correlations between other brain regions of interest (Kuang, Guo, An, Zhao \& He, 2014a). The ROIs exceeding a threshold value, or a significance value defined by performing hypothesis testing for the correlation. Consequently, the method generates a map of the z-score values indicating how those ROIs are correlating with each other. The correlation between ROIs is calculated by the average time series as given in Equation 1, where $C S B$ is the correlation generated through the SBC approach, $S$ is the BOLD time series of the seed and $B$ is the BOLD time series of a selected voxel.

$$
C S B=\frac{\sum_{t=0}^{T} S(t) * B(t)}{\sqrt{\sum_{t=0}^{T} S(t)^{2}}}
$$

The selection of the seed has an impact on the accuracy of the generated network. A change in the selection of seed may result in different types of networks and contribute considerably to the analysis results. Even though the careful seed selection is essential to obtain accurate results, the simplicity and the facileness of the implementation makes this approach outperforms the other techniques such as clustering, PCA and graph-based methods. Hence the SBC method is widely used to generate and explore the Default Mode Network (DMN) in many other neurological disorder identification and analysis studies (Wen et al., 2018; Parida, Dehuri \& Cho, 2015).

\section{Feature Considerations}

Regional Homogeneity (ReHo) is a measurement of brain functionalities based on voxels of the brain regions, that evaluates the homogeneity between the time series values of each voxel and its closest neighbours (Sato, Hoexter, Fujita \& Rohde, 2012). This computation depends on the assumption of, essential and innate brain activities are presented as a group of voxels rather than a single voxel. Kendall's coefficient of concordance (KCC) has been used to evaluate the equivalence between voxels and its nearest neighbours (Omidvarnia et al., 2016). Based on these similarity measurements of voxel's BOLD signals, ReHo provides significant information about regional brain activities within the brain. In order to extract the ReHo features, the KCC is calculated for each brain voxel and maps of voxels are generated using the obtained KCC values and standardized for analysis. As an index for the brain activities, ReHo measures are widely used for the analysis of resting-state fMRI data to measure the activation of brain regions for the identification process of different psychiatric disorders (Zang, Jiang, Lu, He \& Tian, 2004).

Since low fluctuations are significant to obtain the correlation between brain regions and restingstate networks, the magnitude of those fluctuations can vary among subjects and the brain regions. Thus, Fractional Amplitude of Low-Frequency Fluctuations (fALFF) (Zou et al., 2008) is used to quantify the amplitude of those low-frequency oscillations. fALFF is a fraction of the amplitude sum of full frequency range observable in a specified signal. fALFF identifies the variations in amplitude power between subjects and conditions, which is sensitive to the noise. fALFF, as a feature extraction method, specifies a calculation of voxel by voxel of BOLD signal's low frequencies spectral power. Some studies observed that children with ADHD show changes in fALFF compared to controls as they investigated fALFF as a reliable biomarker for ADHD (Sato et al., 2012).

\section{Data Augmentation}

Data augmentation enables generating a massive number of images with the use of the available images applying different transformation techniques (Hussain, Gimenez \& Rubin, 2017), regardless of the rare usage of data augmentation in medical image classification, can increase in specificity and sensitivity. Also, data augmentation techniques provide data consistency justifying their hypothesis of using a fixed duration with a relatively short sequence and having a consistent dimension in data (Mao et al., 2019). 


\section{Related Studies}

EEG and fMRI data have been widely used for the classification process of ADHD based on machine learning and deep learning techniques (De Silva et al., 2019a; Meedeniya \& Rubasinghe, 2020; Lenartowicz \& Loo, 2014). The functionality of the brain structure was effectively reviewed using neuroimages with its temporal and spatial resolution for diagnosis of various psychiatric disorders including ADHD. A study has (Mao et al., 2019) introduced a novel idea based on granular computing for fMRI data which also explored the fusion of both spatial and temporal granularity of fMRI images. The 3D Convolution Neural Network (CNN) was used with spatial feature data from fMRI images and another model of recurrent neural network and feature pooling was developed for comparison of results. Then the proposed solution method was extended to learn spatial and temporal data simultaneously using 4D CNN approach which has obtained high performance.

Many studies have used fALFF, ReHo and independent component analysis (ICA) maps features for the effective understanding of neuroimage data (Song et al., 2011; Zou, Zheng, Miao, Mckeown \& Wang, 2017). Additionally, different combinations of ReHo, Resting-State Networks (RSN) and ALFF have experimented (Miao \& Zhang, 2017). It was identified that ReHo and ALFF, give more classifying information with limited accuracy and the combination of ReHo, ALFF, and RSN, features have shown an accuracy of 67\%. Another study (Hao, Yin \& He, 2015) has used Deep Bayesian Network (BN) by using both Bayesian network and Deep Belief Network (DBN) to execute the dimensionality reduction to extract the features from the relationships and obtained an accuracy of $66.3 \%$ by applying SVM classifier. Multimodal features with multi-kernel learning have also been used for MRI and fMRI data with grey matter probability, cortical thickness and ReHo features (Dai, Wang, Hua \& He, 2012).

Table 1 shows the different learning models used by several related studies. These classification techniques include Support Vector Machine (SVM), Extreme Learning Machine (ELM), Bayesian Network (BN), Deep Belief Network (DBN), Fully Connected Cascade Artificial Neural Network (FCC ANN), Convolutional Neural Network (CNN), Gaussian Naive Bayes (GNB), Linear Discrimination Classifier (LDC) and k - Nearest Neighbour (kNN). Most of the considered studies have used deep learning techniques, due to the capability of learning complex relationships and creating generalize models. Few have applied $\mathrm{kNN}$ as it has the limitations of considering homogeneous features and sensitivity to outliers.

The algorithm of ELM on structural MRI (sMRI) to afford an accurate objective clinical diagnosis to classify ADHD. ELM and SVM learning algorithms were comparatively applied to ADHD classification (Peng et al., 2013) to observe higher accuracy and higher performance among these two learning algorithms. They have shown an accuracy of $90.18 \%$ from the ELM with 0.57 kappa score and nested cross-validation. As an improved version of the feature consideration in fMRI data (Kuang at al.,) was observed with frequency features using the brain activation for each voxel or the brain area of interest. Their work has given promising results and narrowed down the feature space to the brain areas of interest. However, this feature extraction methodology has the inherited imbalance of the DBN in the application of fMRI data (Kuang et al.,2014b).

Neural networks such as ANN, CNN have become a popular feature selection method for medical data (Meedeniya \& Rubasinghe, 2020). They perform well in the domain of the medicinal imaging analysis, rather than the conventional feature selection techniques such as DWFS, FDT, and FCBFS. A recent study (Meraj, Yaakob, Azman, Rum \& Nazri, 2019) shows that CNN outperforms the other machine learning techniques such as multi-layer neural networks, decision trees, fuzzy logic, and genetic algorithms. A classification method based on Artificial Neural Network (ANN) for distinguishing ADHD from normal groups and its subtypes has been proposed (Deshpande et al., 2015). Several neural network topologies were compared to enhance the classification capability and to obtain the best architecture with broad generalization ability to perform well. Compared to the Multilayer Perceptron (MLP), the Single Hidden Layer (SHL) has overcome the difficulties in 
Table 1. Related work in learning models

\begin{tabular}{|l|c|c|c|c|c|c|c|c|c|}
\hline $\begin{array}{l}\text { Technique } \\
\text { Related work }\end{array}$ & SVM & ELM & BN & DBN & $\begin{array}{c}\text { FCC } \\
\text { ANN }\end{array}$ & CNN & GNB & LDC & kNN \\
\hline ELM based classification of ADHD (Peng et al., 2013) & $\mathrm{X}$ & $\mathrm{X}$ & & & & & & & \\
\hline 4D CNN Based ADHD classification (Mao et al., 2019) & & & & & & $\mathrm{X}$ & & & \\
\hline ADHD classification (Kuang et al., 2014a; Kuang et al.,2014b) & & & & $\mathrm{X}$ & & & & & \\
\hline $\begin{array}{l}\text { Discrimination of ADHD using Deep Bayesian Network (Hao, } \\
\text { Yin \& He, 2015) }\end{array}$ & & & $\mathrm{X}$ & & & & & & \\
\hline $\begin{array}{l}\text { FCC ANN for ADHD classification (Deshpande, Wang, } \\
\text { Rangaprakash \& Wilamowski, 2015) }\end{array}$ & $\mathrm{X}$ & & & & $\mathrm{X}$ & & & & \\
\hline ADHD prediction (Sato et. al, 2012) & $\mathrm{X}$ & & & & & & & & \\
\hline $\begin{array}{l}\text { ADHD classification using fMRI (Rubasinghe \& Meedeniya, } \\
\text { 2020) }\end{array}$ & $\mathrm{X}$ & & & & & & $\mathrm{X}$ & & $\mathrm{X}$ \\
\hline ADHD classification with SBC (Ariyarathne et al., 2020) & & & & & & $\mathrm{X}$ & & & \\
\hline
\end{tabular}

MLP with the use of a nonlinear mapping system. They have shown the accuracy of $90 \%$ and $95 \%$ between ADHD and normal groups and between ADHD subtypes respectively, using FCC ANN.

A previous study has used an fMRI dataset, which is already preprocessed using C-PAC software pipeline to show the effect of DMN regions for ADHD classification (Ariyarathne et al., 2020). This study has extracted features based on SBC, that measure the functional connectivity among the seeds and other brain voxels. They have considered the DMN regions Posterior Cingulate cortex (PCC), Left Temporoparietal junction (LTJ), Right Temporoparietal junction (RTJ), Medial Prefrontal Cortex (MPC). The CNN based classification has shown accuracies in the range of $84 \%$ $86 \%$ for different DMN regions. Further, another approach for ADHD classification using fMRI is presented in (Rubasinghe \& Meedeniya, 2020). This work has also used the functional connectivity measures to the obtain correlation between the brain regions. They have considered two datasets, one for ADHD and the other for ASD (Autism Spectrum Disorder) and evaluated the classification results using a set of learning models. Mainly, they have shown the need for a generic neuroscience decision support system to support the diagnosis process with different classification techniques and diverse fMRI datasets.

\section{SYSTEM DESIGN}

The system of ADHD identification has been designed by using fMRI data from the ADHD 200-Global Competition (Bellec et al., 2017). The overview of the proposed system ADHD-Care_v2 is shown in Figure 1. Normalization, band-pass filtering, motion correction, co-registration, and slice timing correction are the techniques that are used to preprocess the fMRI data (De Silva et al., 2019a; Meedeniya \& Rubasinghe, 2020). Spatial normalization is applied to identify the differences between fMRI data by comparing the image volumes. The recognized variations of shapes are minimized by wrapping, squeezing and stretching images of the brain for motion correction using SPM12 tool. In some images, the position of the brain may be imprecise when head motion happens. Thus, with the use of co-regression to align brain images spatially, motion correction adjusts the position of the images. To reduce time variations between slices as there can be delays in hundreds of milliseconds between acquired slices, slice time correction is used.

The feature extraction was done by using this preprocessed data. The SBC is used as the feature extraction and selection mechanism. The features of ReHo and fALFF were taken to the process of feature extraction and selection in related studies (Zou et al., 2017), which obtained a significant accuracy. Thus, this study also considered ReHo and fALFF features for the classification process 
Figure 1. System overview of ADHD-Care_v2

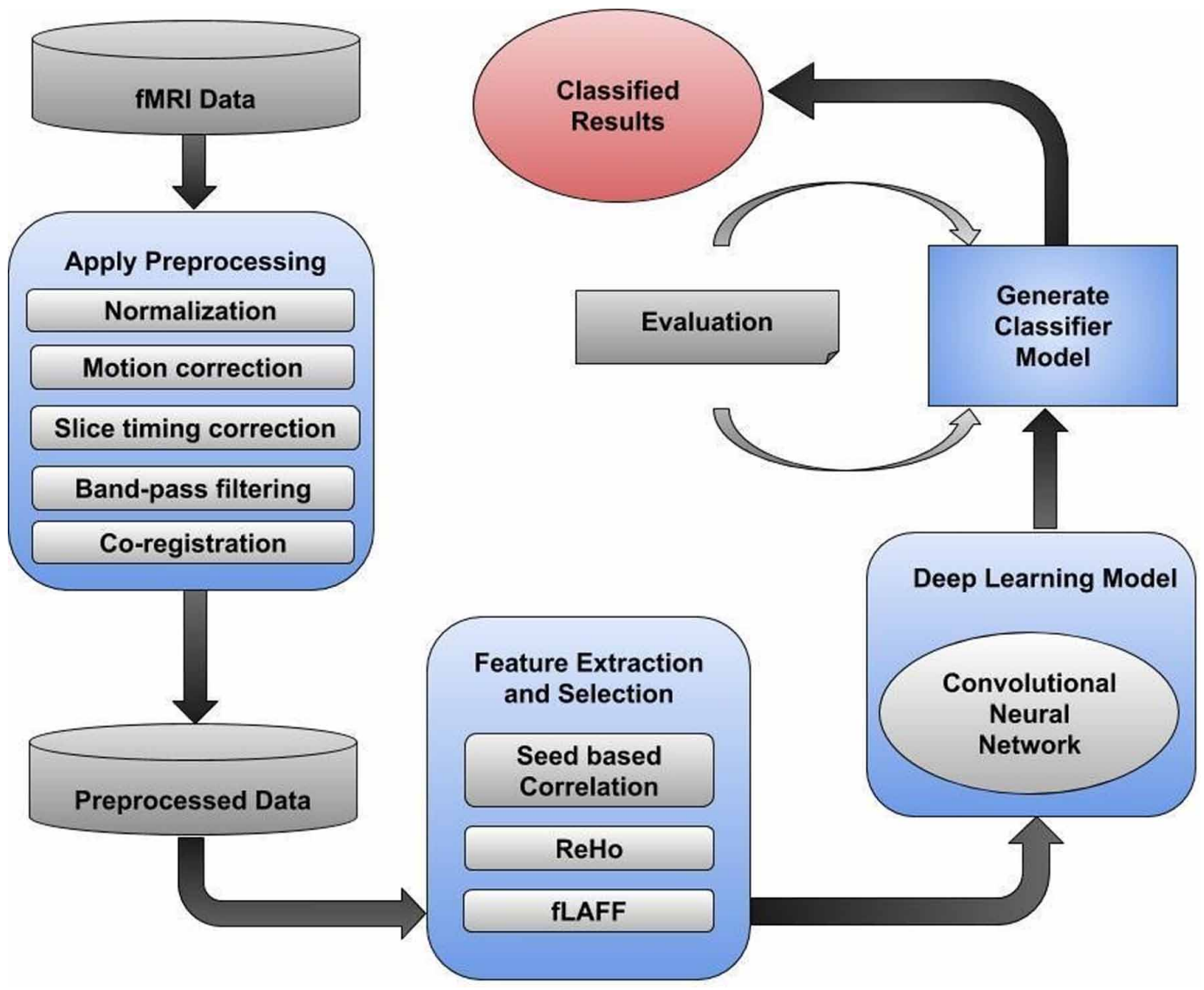

of ADHD. Since fMRI images are in 4D format, off-the-shelf CNN architectures cannot be used for 4D data. After the feature extraction process, within the deep learning model, the processed 4D fMRI images are transformed into 2D images. Also, data augmentation is performed to increase the size of the dataset. To generate a deep learning model to classify ADHD, CNN has been used as the classifier in our study.

Further, model evaluation techniques were applied to achieve acceptable accuracy. The accuracy is calculated for the generated models as the evaluation is done with different neural networks which consist of hidden and fully connected layers with various activation functions. In order to obtain high accuracy, some image processing methods are yet to be used to normalize the image and reduce the dimensionality as the dataset has high dimensionality. Finally, the classifier model was generated after several evaluations to accurately identify ADHD with the fMRI data.

\section{METHODOLOGY}

\section{Materials}

This study has used two fMRI datasets that are released by the ADHD-200 Global Competition from the Neuro Bureau (Bellec et al., 2017). The dataset is based on 40 participants between the ages of $8-21$ from 4 data centres namely, New York University (NYU), Peking University (Peking-1) and Kennedy Krieger Institute (KKI), Oregon Health and Science University (OHSU) and University 
of Pittsburgh data centres. The total number of fMRI images in each dataset is 40 , that contains 10 participant data from each of the 4 data centres. Thus, both datasets represent the data of the same set of participants and obtained from the same data centres. The dataset was categorized into two main groups with 20 ADHD positive subjects and 20 healthy subjects. The first dataset, D1, consists of un-processed raw 4D fMRI data. The second dataset, D2, is the corresponding pre-processed restingstate fMRI data using Configurable Pipeline for the Analysis of Connectomes (C-PAC) open-source software pipeline (Ashburner et al., 2014). The dataset D2 has already preprocessed using slice time correction, intensity normalization, temporal filtering, spatial smoothing, motion scrubbing, volume realignment, skull stripping and functional co-registration. D2 was extracted using Echo Planar Images (EPI), which is a fast technique to acquire images.

Both preprocessed $404 \mathrm{D}$ fMRI datasets are converted into 2D images before the learning process to comply with the $2 \mathrm{D} \mathrm{CNN}$. In this process, each 4D fMRI image is normalized to generate 60 slices as 2D images using med2image python library. The data augmentation process is based on $2400 \mathrm{fMRI}$ images. Then, with the 3 types of augmentation techniques, the dataset is increased up to 7200 images, which is the input to the CNN model. The visualization of these fMRI images is shown in Figure 2. The first row shows a data instance for ADHD positive and the second row shows an instance of non-ADHD subjects. During this study, the dataset D1 is preprocessed using the SMP12 software application. The results obtained using the dataset D2, are used to validate the proposed preprocessing approach with the raw dataset D1 and to identify the optimal feature extraction methods such as SBC, ReHo and fALFF.

\section{Data Pre-Processing}

The raw images of fMRI data were preprocessed by applying realignment, slice time correction, normalization, coregistration and smoothing techniques (De Silva et al., 2019a; Meedeniya \& Rubasinghe, 2020). The preprocessing was supported by SPM12 software for the performance of different spatial functionalities. SPM12 is free, open-source software that is supported by MATLAB background for the implementation of Statistical Parametric Mapping which is used to analyse the spatially extended processes.

Initially, the fMRI images were realigned to match each image to maintain the consistency and adhere to a brain image template by transforming and rotating the images. As subjects tend to move during the scanning process, it is possible to have different movement artefacts. This may cause various locational mismatches in time-series images. Also, these mismatches will add extra noise to the image data by reducing its sensitivity. Transformations are done by minimizing the squared differences between images which reduces a cost function.

Slice time correction was performed by aligning the data and shifting its signal phase by linear interpolation given in Equation 2. This makes the relevant data on each slice correspond to the same time point where $X_{t}^{\text {new }}$ refers to the corrected slice time at time $t, \Delta$ refers to the change of the correction. It is performed by shifting each voxel's time series to appear as they were acquired simultaneously. As each slice in the image volume is not acquired at the same time, the time differences may occur within the order of acquisition of slices. The slice time correction adjusts the image values to make all the brain voxels to appear as they were processed in the same time duration.

$$
X_{t}^{\text {new }}=(1-\Delta)\left(X_{t}-1\right)\left(\Delta X_{t}\right)
$$

The brain images were normalized to reduce the impact of the shape effect of the brain which varies from one subject to another. Each volume of the fMRI images was compared to identify the differences. In the normalization process, each brain image is adjusted according to a template brain image of the first subject anatomically which ensures that the spatial locations are consistent across 


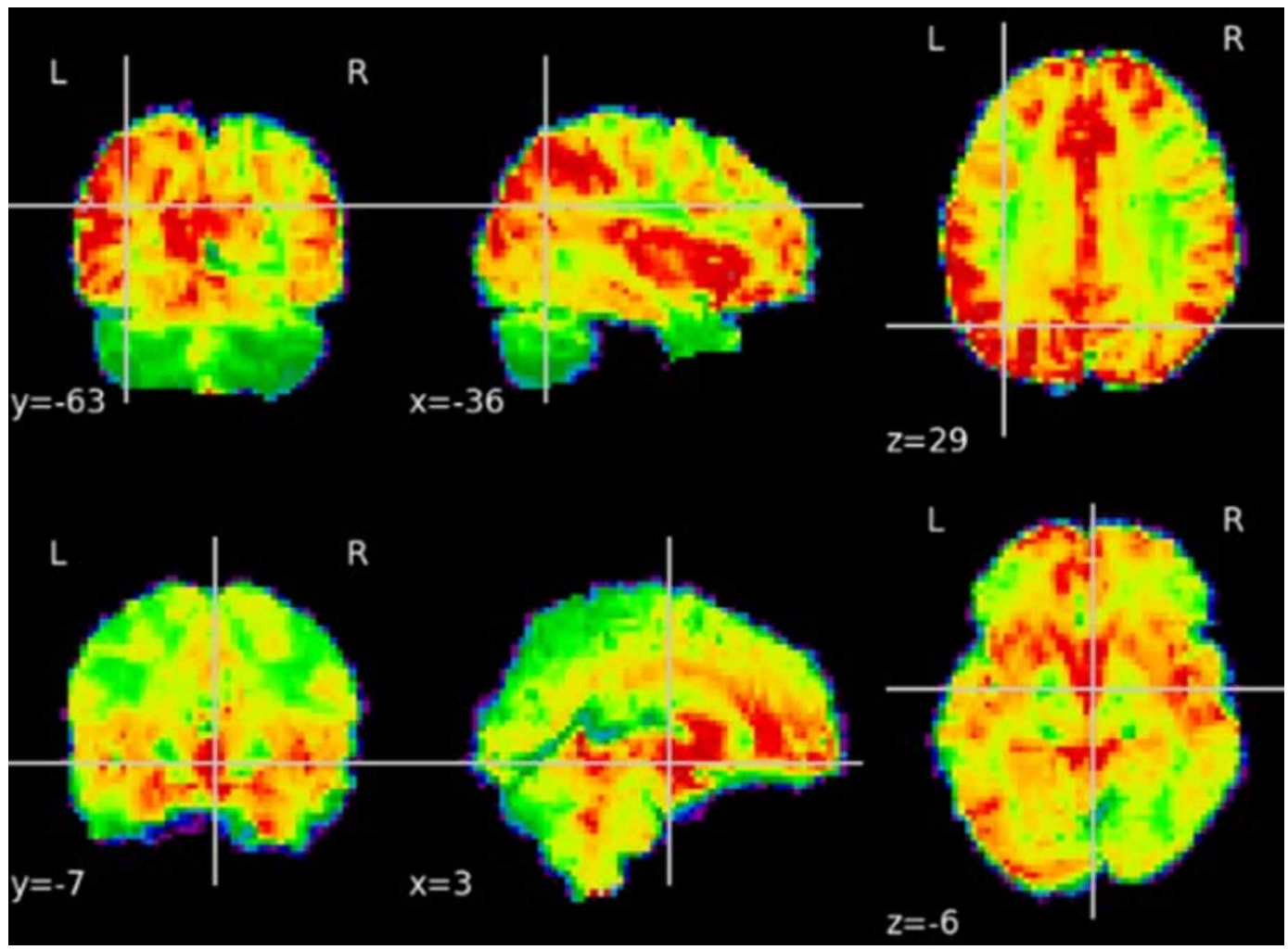

the entire dataset. Noise reduction, extraction and the segmentation of the brain, correction of bias have also performed on fMRI brain images under image normalization. Various tissue sections were sectioned for segmentation and the varying intensity dissimilarities were removed under bias correction. The images were coregistered using the same subject using image interpolations and transformations. This process enables aligning structural and functional images correctly which is effective in low-resolution images where anatomical details are given.

Finally, the processed images were smoothed by increasing the signal-to-noise ratio and averaging of inter-subjects, by applying a Gaussian kernel and convolving the image with the kernel. This further removes anatomical differences between images by averaging the brain voxel with the weighted sums of its closest voxels. The impact of denoising techniques using SPM12 preprocessing for the classification accuracy is given in Table 2, in the Evaluation Section.

\section{Feature Extraction}

The feature extraction process is used to derive informative values and reduce the redundancies prior to the use of learning models. This approach considers three main features: fALFF, ReHo and SBC, for fMRI feature extraction. The connectivity in DMN brain regions is extracted using SBC, which is an effective method to identify the functional connectivity of the brain by measuring the correlation of time series of different brain regions. We have used the raw data obtained from the Neuro Bureau (Bellec et al., 2017), which is then preprocessed using SPM12, and fed for the feature selection process. Additionally, we have used the already preprocessed data of the same, obtained from C-PAC, for the validation process. 


\section{SBC Feature Extraction}

First, the SBC features are extraction process is described as follows. Here, the pre-processed data is used to calculate the correlations considering the DMN regions as the seeds separately, using SPM12 software. The considered DMN regions include the Posterior Cingulate cortex (PCC), Left Temporoparietal junction (LT), Right Temporoparietal junction (RT), Medial Prefrontal Cortex (MPC). The fMRI BOLD signals are subjected to the application of a region radius of $8 \mathrm{~mm}$, a low pass filter of $0.1 \mathrm{~Hz}$, high pass filter of $0.01 \mathrm{~Hz}$ to reduce the noise and clean the data. These frequency ranges are selected, as the smaller high or low pass filters reduce the noise, further. This mask extracts time series from the fMRI data to generate the correlations.

The algorithm for SBC calculation, considering the entire DMN region is shown in Algorithm 1. In order to calculate the seed-based correlation, the pre-processed resting-state fMRI dataset has been used as the inputs and the algorithm returns the calculated correlation values based on the defined seed regions of the considered brain regions. Each of the data samples is iterated through this algorithm starting by extracting functional (line No 4) and confounds (line No 5) from the data sample. The functional data refers to the BOLD signal level in each of the voxels, which serves as the main data contribution for the analysis. Confounds refer to non-neural fluctuations induced by the environmental factors such as head motion, equipment errors, noise etc. for each subject's fMRI images.

In order to further correct the pre-processed data during the correlation calculation, the confounds are considered. The regions of interest have been chosen as the DMN, where the ADHD subjects have shown significant-high activity compared to the control groups (Metin et al., 2015). Furthermore, based on the related studies (Uddin et al., 2008), PCC, MPC, RTJ, LTJ region coordinates have been used as the seeds in the DMN region (line 6). Thus, these DMN region coordinates are considered as the seeds for the correlation extraction between the seed region and remaining brain areas. The coordinates were mapped into the MNI space. Then a seed brain masker is created using the defined seeds (line 7). In this study, a radius of $8 \mathrm{~mm}$ has been used as empirical results suggested. Thus, the seed masker, which summarizes seed time series values is used to extract time-series data from fMRI images within a considered sphere area, according to the given radius to a value of $8 \mathrm{~mm}$.

\section{Function: generate SBC array ()}

2. dataset $=$ adhd_pre-processed_dataset

3. for $\mathrm{i}$ in range (dataset_size):

4. functional_data $=$ dataset.functionaldata $($ )

5. $\quad$ confound_data $=$ dataset.confounds ()

6. dmn_coordinate $=[(\mathrm{x}, \mathrm{y}, \mathrm{z})]$

7. seed_masker(dmn_coordinates, radius)

8. seed_time_series = seed_masker.transform(functional_data,confound_data)

9. brain_masker ()

10. brain_time_series = brain_masker.transform (functional_data, confound_data)

11. seed_based_correlation $=$ product(seed_time_series,brain_time_series)

12. seed_based_correlation_fisher_z $=$ arc_tan(seed_based_correlation)

13. seed_based_correlation_img = brain_masker.inverse_transform(seed_based_correlations)

Then for each of the data samples, the seed brain mask is applied and extracted the data in that DMN region from the voxel-wide time series of the pre-processed data. This is used to calculate the seed-based correlation in a later stage (line 11). Apart from the seed masker, a whole-brain masker is defined to capture the brain data in a normalized manner (line 9). The mean values for the derived time series data are extracted for DMN regions after removing the confound values. 
The time series of each of these masks are used to calculate the correlation, where fMRI represents a temporal behaviour, which is well-expressed by a time series. The same procedure is followed to extract a brain-wide time series. Next, the seed-based correlation has been calculated by taking the dot product of these time series (line 11), such that correlating the seed time series values with each brain voxel signals. A fisher-z transformation is applied to normalize the obtained correlation values. The derived normally distributed data and the correlations are stored as a new fMRI, which then directly fed into CNN. As brain imaging is used for the classification supported by a CNN, the normalized correlation values have been converted to neuroimages applying the brain mask (line 13). This process prepares the pre-processed data and extracts the features selected by the areas of interest of the brain.

Moreover, the proposed algorithm analyses the underlying time series produced in fMRI data than the other available methodologies. Since several masking approaches have been used, the functionality is useful, compared to the other hand-crafted features like fALFF, ALFF, ReHo. Further, this could be improved by considering different regions along with the DMN region, by supporting multi-regional seed-based correlation calculation to infer more improved results.

A sample of the correlation images is shown in Figure 3.

\section{Figure 3. Seed -based correlation of PCC region}
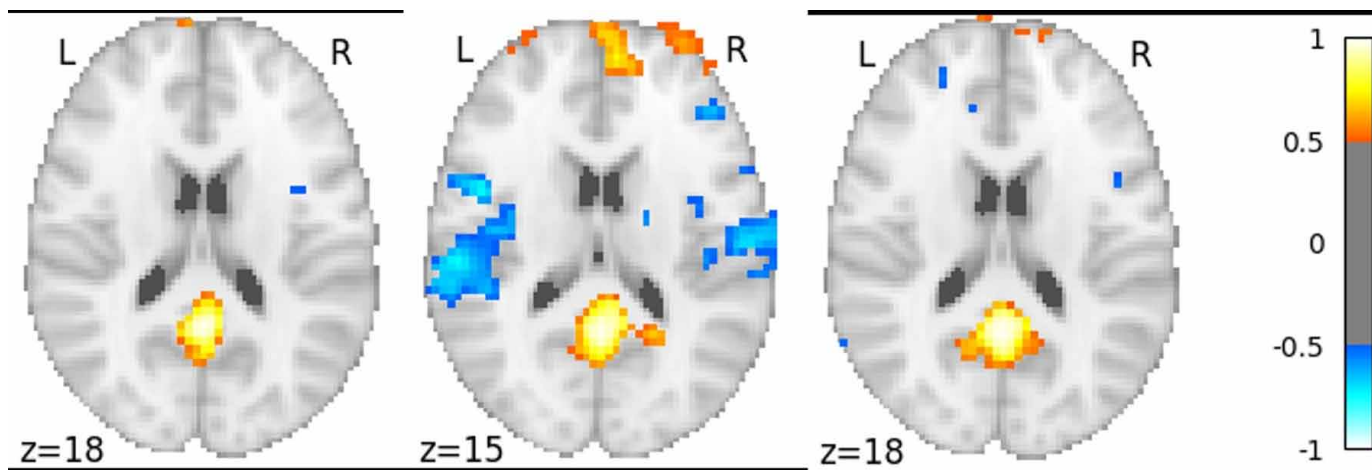

\section{ReHo Feature Extraction}

Next, the Kendall coefficient of concordance is used for the ReHo feature extraction, by measuring the brain activity using fMRI data. It is a purifying method for the clusters found in the brain, followed by an approach similar to the k-nearest neighbour method, where the similarity is measured by the Kendall coefficient, as given in Equation 3. This method measures the similarity of a set of BOLD signals, which generates a similarity matrix as a map. The usage of ReHo maps in the clinical practice has been accepted including ADHD diagnosis (Zou et al., 2008).

$$
W=\frac{\sum_{i=0}^{n}\left(R_{i}\right)^{2}-n(\underline{R})^{2}}{\frac{1}{12} K^{2}\left(n^{3}-n\right)}
$$


where $R_{i}$ is the rank of the BOLD signal, $\underline{\mathrm{R}}$ is the mean of the time series calculated by $\underline{\mathrm{R}}=((\mathrm{n}+1)$ $\mathrm{K}) / 2$ to which the voxel belongs, $\mathrm{K}$ is the number of time series measured in the considered cluster and $\mathrm{n}$ is the number of ranks.

\section{fALFF Feature Extraction}

To extract fALFF features, the BOLD signal is converted to the frequency domain without any bandpass filter and calculate the square root of each frequency of the power spectrum, which is sensitive to the noise. Then the summation of the low-frequency range $(0.01-0.08 \mathrm{~Hz})$ is calculated and divided by the entire range, to extract fALFF features. The corresponding ReHo maps and fALFF maps for the already pre-processed data are available from the ADHD-200 competition repository in the Neuro Benro website (Bellec et al., 2017), which are used to validate this approach in Section 5.

\section{Data Augmentation}

The deployment of a deep learning model requires a sufficient amount of data. Thus, data augmentation is applied for the 2400 2D images, by applying the three techniques: random rotation, height shift augmentation, and horizontal flip techniques ("Image Preprocessing - Keras Documentation", 2019). We have used the python library Keras for data augmentation. Since the 2D images are generated considering a fixed duration for each of the subjects, the consistency of the data is preserved with the application of data augmentation.

The results of the data augmentation methods are shown in Figure 4. The visualization of augmented images describes the application of random rotation, height shift of the original images along with horizontal flipping. This enables increased data size and having exposure to the combination of different types of images that may appear in the training dataset.

Figure 4. Data augmentation techniques applied

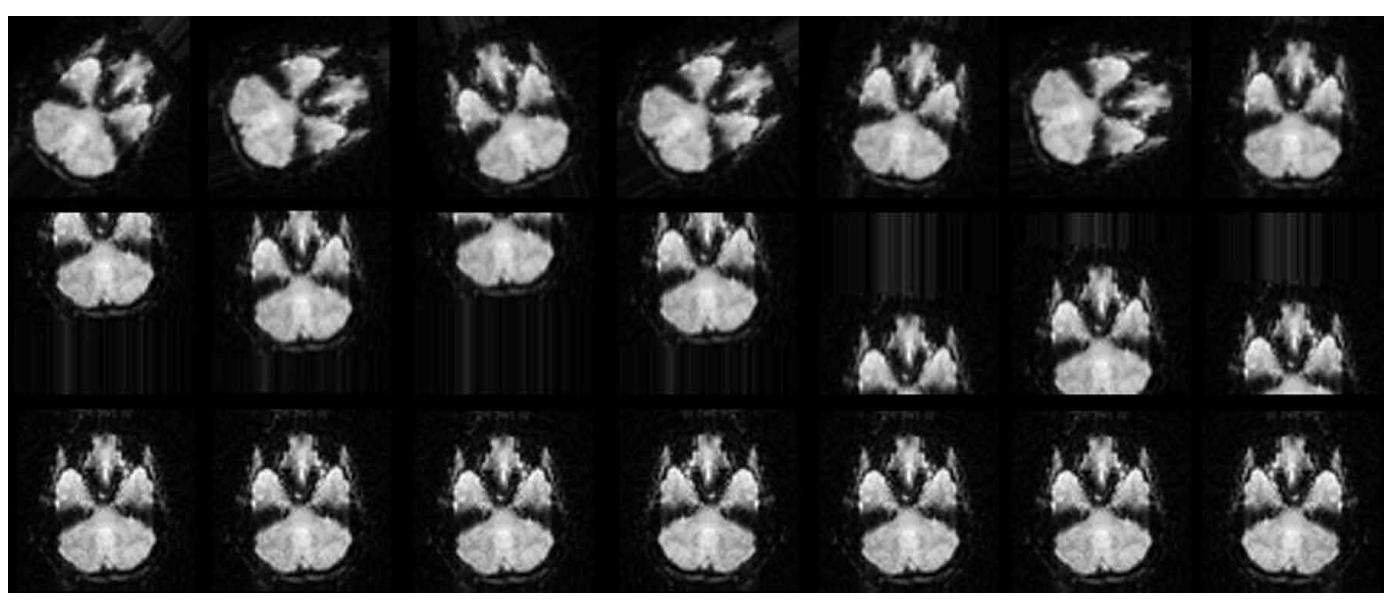

Apart from the basic augmentation methods, prior to the application to the constructed CNN model, few other advanced data augmentation techniques were applied including rescaling and zooming. The rescaling is applied to normalize the colour range of the images into 0 to 1 from 0 to 255 scale, which becomes too high for the model to handle. Zooming is applied to change the aspect ratio of the image providing more variety of images. These techniques are performed during the learning model to prevent overfitting of the model. In this study, data augmentation methods 
have been applied to prevent overfitting. This technique also enables the data consistency over the training and testing datasets.

\section{Convolutional Neural Network}

A neural network is generated with a convolution layer with a kernel size of $3 * 3$ to support the extraction of local features in the brain images during the training. A smaller kernel size $3 * 3$ was used since the obtained 2D images were smaller in size. This enables extracting local features efficiently. The original images are of size $64 * 64$ with 32 feature maps in each of the 8 layers. To capture the complex, smaller features in each layer, with high accuracy from the learning model, we have used 32 filters to enhance the training that enables generating 32 features maps.

As the activation function, rectified linear unit, which is denoted as 'Relu' is used. Relu is used in the intermediate layers of the $\mathrm{CNN}$, since it consumes less time to compute and eventually converges. With the similar specification of the first layer and 64 filters, the second layer is created and a max-pooling layer of $2 * 2$ pool size is added. A dropout rate of 0.25 was used to flatten and regularize the network model. Here, it is used a smaller dropout rate compared to other layers, since it is less effective for the result and essential in the feature map generation.

The fifth layer is a dense layer of 128 units which will change the dimensions of the vector complying to the result. This is used to finalize the feature maps followed by the 'Relu' activation function. Here, the feature maps are completed, and a high dropout rate will not highly impact the classification result. Thus, to avoid overfitting, a dropout rate of 0.5 is added.

Then, 2 units of the dense layers are added to enable the classification. Since this stage is closer to the final stage, it is not necessary to apply more dense layers. Finally, the neural network is wrapped with the softmax activation function was added to select the most probable value from the generated probability values by the CNN. The architecture is shown in Figure 5 and Figure 6.

Although such a deep neural network can extract features by itself, the visualization of those features is unpredictable. It affects the consistency of the feature set since the images are generated in the $2 \mathrm{D}$ format for each volume based on the correlation. Thus, it promises the consistency and coherence of the features, while providing visualization capabilities.

A learning model aims to minimize the difference between the predictions and the actual result, which is denoted as the cost function of a model. Generally, the result of a model depends on the (1) learning rate, (2) the number of images and (3) the validation technique used. The iterative machine

\section{Figure 5. Convolutional neural network architecture}

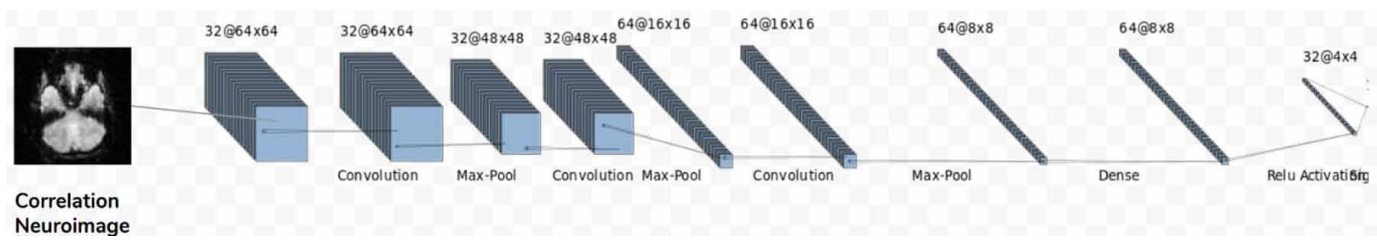

Figure 6. Convolutional neural network modular architecture

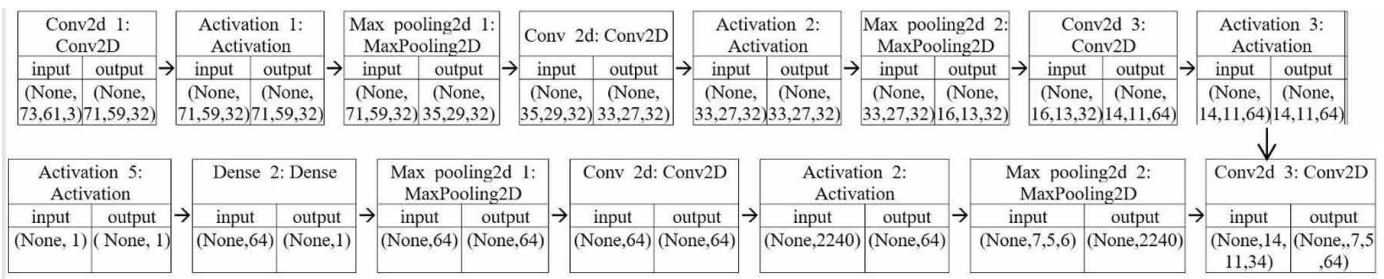


learning algorithm, gradient descent with appropriate optimizer supports to achieve this aim of the neural network. Accordingly, to enable fast training and optimized learning, an adaptive learning rate is derived from the RMSProp optimization algorithm (Ruder, 2016). Thus, gradient descent supports a generalized algorithm to optimize the weights and minimize the cost function, hence better prediction accuracy.

As the validation technique for the model, the train-test split of 2:1 as used. Moreover, a validation generator is used to split the training data further as 0.2 from the training set. This validation dataset is populated before the shuffling of training data. The importance of this validation dataset is that after each epoch, it will use this validation dataset for evaluating the loss and accuracy of the model. This prevents further overfitting that would be caused by adjustments of weights in the training time.

\section{RESULT EVALUATION}

\section{Experiment Setup and Result Analysis}

This aim of this study is to identify fMRI data pre-processing and feature extraction techniques, that will result in high classification accuracy using CNN. We have defined several process combinations to compare the results obtained from the un-processed fMRI data using SPM12 and the corresponding pre-processed data using C-PAC. Recall from the materials subsection, the dataset D1, consists of un-processed raw 4D fMRI data and the dataset D2, is the corresponding pre-processed resting-state fMRI data using C-PAC. Also, the evaluation includes the classification accuracy comparison based on different feature extraction methods including SBC, fALFF and ReHo. We have considered process combinations to emphasise the need for pre-processing and decide the optimal feature extraction methods, as follows:

Task 1- Take D1, unprocessed raw fMRI images as the input and pre-process using SPM12, feature extraction with SBC and then perform CNN

Task 2- Take D2, pre-processed fMRI images using C-PAC as the input and apply SBC and then perform CNN

Task 3- Take D1, unprocessed raw fMRI images as the input and pre-process using SPM12, feature extraction with both ReHo and fALFF, without SBC and then perform CNN

Task 4- Take D1, unprocessed raw fMRI images as the input and pre-process using SPM12, feature extraction with only ReHo and then perform CNN

Task 5- Task 4- Take D1, unprocessed raw fMRI images as the input and pre-process using SPM12, feature extraction with the only fALFF and then perform CNN

Task 6- Take D1, unprocessed raw fMRI images as the input and pre-process using SPM12, feature extraction with all three types and then perform $\mathrm{CNN}$

Task 7- Take D1, unprocessed raw fMRI images as the input and pre-process using SPM12, feature extraction with both SBC and ReHo, and then perform CNN

Task 8- Take D1, unprocessed raw fMRI images as the input and pre-process using SPM12, feature extraction with both SBCand fALFF, and then perform CNN

Task 9- Take D1, unprocessed raw fMRI images as the input and directly perform CNN, without pre-processing or feature extraction

The metrics of the effectiveness of each of the process combinations are obtained by the classification results generated through the CNN model. Hence to obtain accurate results, $33 \%$ of the entire dataset for each of these categories was randomly chosen as testing data for each iteration. The evaluation metrics were selected as accuracy, specificity, and sensitivity. The accuracy was measured with the test set separated earlier supported by the validation set during the training period. Sensitivity and specificity are two important measures in medical applications. The sensitivity also 
referred to as the recall, which is the proportion of actual ADHD positives identified among the actual ADHD population. The specificity measures the proportion of correctly identified healthy subjects in the actual healthy population. The derivation equations for specificity and sensitivity are shown in Equation 5 and Equation 6, respectively.

$$
\text { Sensitivity }=\frac{\text { True Positives }}{\text { TruePositives }+ \text { False Negatives }}
$$

$$
\text { Specificity }=\frac{\text { True Negatives }}{\text { True Negatives }+ \text { False Positives }}
$$

As shown in Table 2 and Figure 7, the pre-processing from SPM12 has achieved 85\% accuracy and sensitivity of $72.80 \%$ proving the high pre-processing quality and the effective feature extraction using the SBC technique. The tasks are ordered based on the obtained accuracy values, for a clear understanding. For the evaluation purposes, we have selected the highest accuracy obtained for considered DMN regions.

Considering the selection of the pre-processing tools, the values obtained with both C-PAC and SPM12, are in a similar rage. That confirms that these tools are competent in their pre-processing methodologies. Moreover, Task 9, the process without pre-processing and feature extraction methods, has given the minimum accuracy values. Also, the comparative sensitivity value of the SBC approach is high. This shows the applicability in effective identification of the ADHD positive subjects. In another point of view, the other selected feature extraction methods, fALFF and ReHo, have shown an average classification accuracy values along with specificity and sensitivity metrics in the same range. Although, fALFF and ReHo maps are used as prominent features in extensive research (Kuang et al., 2014a; Sato et al., 2012), the results in Table 2 shows the effectiveness of SBC in the feature extraction process.

Compared to Task 1, which has used the SBC technique, the process combination tasks with fALFF and ReHo feature extraction techniques have shown lower accuracy values. One possible reason for having low values could be that, these features are extracted considering the whole-brain

\begin{tabular}{|c|c|c|c|c|c|c|c|c|c|}
\hline \multirow[t]{3}{*}{ Task ID } & \multirow[t]{3}{*}{ Dataset } & \multicolumn{5}{|c|}{ Processing technique } & & & \\
\hline & & \multirow[t]{2}{*}{ SPM12 } & \multicolumn{3}{|c|}{ Feature extraction } & \multirow[t]{2}{*}{ CNN } & & & \multirow[b]{2}{*}{ Sensitivity } \\
\hline & & & SBC & ReHo & fALFF & & Accuracy & Specificity & \\
\hline $\mathrm{T} 1$ & D1 & $\mathrm{X}$ & $\mathrm{x}$ & - & - & $\mathrm{X}$ & $85.36 \%$ & $66.54 \%$ & $72.80 \%$ \\
\hline $\mathrm{T} 2$ & D2 & - & $\mathrm{X}$ & - & - & $\mathrm{x}$ & $83.40 \%$ & $66.78 \%$ & $70.22 \%$ \\
\hline $\mathrm{T} 3$ & D1 & $\mathrm{X}$ & - & $\mathrm{X}$ & $\mathrm{X}$ & $\mathrm{x}$ & $71.79 \%$ & $63.22 \%$ & $68.25 \%$ \\
\hline $\mathrm{T} 4$ & D1 & $\mathrm{x}$ & - & $\mathrm{X}$ & - & $\mathrm{X}$ & $67.30 \%$ & $54.19 \%$ & $60.22 \%$ \\
\hline T5 & D1 & $\mathrm{X}$ & - & - & $\mathrm{X}$ & $\mathrm{X}$ & $67.17 \%$ & $55.22 \%$ & $60.24 \%$ \\
\hline T6 & D1 & $\mathrm{X}$ & $\mathrm{x}$ & $\mathrm{X}$ & $\mathrm{X}$ & $\mathrm{x}$ & $67.12 \%$ & $61.67 \%$ & $65.53 \%$ \\
\hline $\mathrm{T} 7$ & D1 & $\mathrm{X}$ & $\mathrm{x}$ & $\mathrm{x}$ & - & $\mathrm{x}$ & $66.75 \%$ & $61.22 \%$ & $63.42 \%$ \\
\hline $\mathrm{T} 8$ & D1 & $\mathrm{x}$ & $\mathrm{X}$ & - & $\mathrm{X}$ & $\mathrm{x}$ & $64.75 \%$ & $57.16 \%$ & $59.35 \%$ \\
\hline T9 & D1 & - & - & - & - & $\mathrm{X}$ & $57.30 \%$ & $49.88 \%$ & $50.23 \%$ \\
\hline
\end{tabular}

Table 2. Processing results for different combinations 


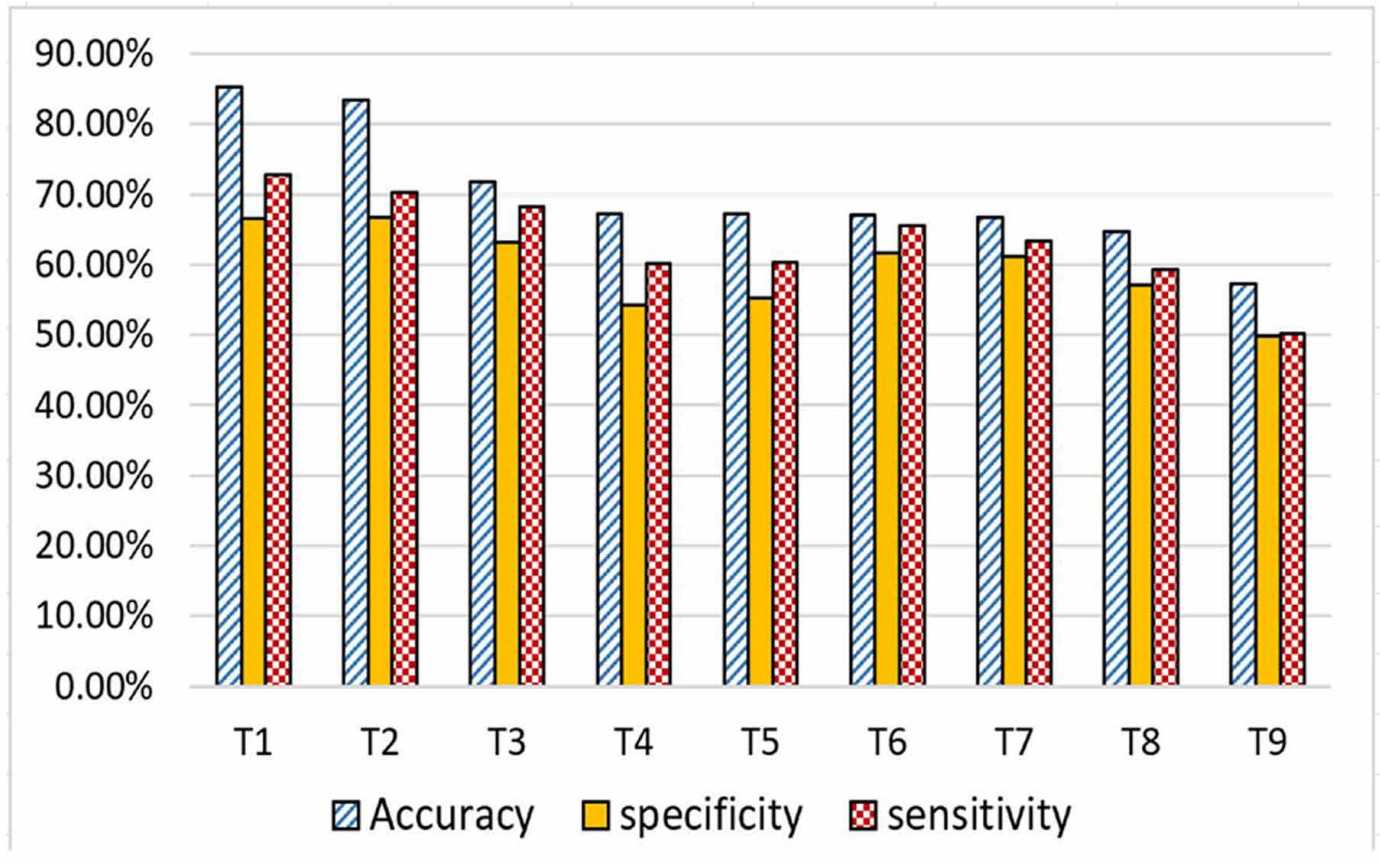

perspective, whereas SBC is generated by focusing the DMN region that it has found (Metin et al., 2015). Since the combination of fALFF and ReHo features are based on functional connectivity, it can derive the actual brain dynamics constructively. These combination tasks have shown a similar accuracy of $67 \%$ and a sensitivity of $60 \%$. Although these results are approximately confirmed by the related study (Sato et al., 2012), the results obtained from this study have achieved comparatively higher accuracy. Moreover, the careful design of CNN, prevention of overfitting images using data augmentation techniques, having mechanisms to handle variance and bias influenced by different data sources have led to obtaining higher accuracy values.

Further, the combination of fALFF and ReHo features have shown better accuracy values compared to fALFF and ReHo when considered separately. This implied that the combination of fALFF and ReHo can provide higher discriminative value than considered separately. Comparing the accuracy values of all the feature combinations, the SBC approach has exclusively performed better than other considered combinations.

\section{Analysis of Feature Extraction Process}

In order to calculate the correlation between the selected features of fALFF, ReHo, and SBC, statistical techniques such as spatial distribution analysis (Cortese et al., 2012) and across-subject analysis (Penberthy et al, 2005) have been used. This process was suggested for the identification of the relationship between metabolism and resting-state fMRI data (Aiello et al.). As the initial step of the spatial distribution analysis, the obtained feature maps were normalized using Spearman's correlation coefficient (Xiao, Ye, Esteves, \& Rong, 2016), and then calculated the means of each feature voxelwise. The applied equations of Spearman's correlation and the mean maps are listed in Equation 4 


$$
\rho=\frac{\sum_{i=0}^{N}\left(x_{i}-\underline{x}\right)\left(y_{i}-\underline{y}\right)}{\sqrt{\sum_{i=0}^{N}\left(x_{i}-\underline{x}\right)^{2}\left(y_{i}-\underline{y}\right)^{2}}}
$$

Where, $\underline{x}$ refers to the mean of the feature value of all brain voxels and $x_{i}$ refers to the feature value for the voxel $\mathrm{i}$.

Since this spatial distribution can introduce a bias generated by the subject variation, another statistical technique was used to improve the obtained correlation values between features. The across-subjects analysis was performed, and multimodal correlation was calculated by considering the Spearman's correlation coefficient and the mean maps defined by the spatial distribution. We have correlated and analysed the results of features selected by the three algorithms SBC, ReHo, and fALFF. The obtained correlation results are shown in Figure 8.

As the calculated correlation results portray, the correlation between fALFF and ReHo is the highest. In turn, it means that fALFF and ReHo behave in the same way considering the whole-brain connectivity. On the other hand, SBC considering the regional connectivity specifically has been correlated with fALFF and ReHo features lesser. Further, SBC and ReHo have a higher correlation compared to SBC and fALFF, since both features have focused on the regional activity to a certain extent. In the ReHo features, all regions of the brain have been studied while SBC has narrowed down its focus into the areas where ADHD subjects have reported high activity. This correlation outcome has been illustrated and verified in the same manner in the obtained results in the application of CNN.

\section{Result Comparison with Different Classification Algorithms}

Since fMRI data consists of a set of neuroimages, it is not always possible to use classification algorithms such as decision tree, random forest, J48. Although some of the related studies have applied these learning models for the classification process, they have used non-neuro imaging datasets such as questionnaire data or other clinical informative (Brihadiswaran et al., 2019; Meedeniya \& Rubasinghe, 2020; Haputhanthri et al, 2019). Thus, we have compared the proposed CNN architecture with other existing pre-trained CNN models, as shown in Table 3.

In this study, we have considered the converted seed-based featured images from 4D to 2D image data set for classification purposes, which is based on the un-processed dataset D1. Therefore, the CNN based deep learning approach was used for the classification of neuroimages to recognize the patterns of changes in BOLD signal in different DMN regions. The CNN model was designed with 7 layers including fully connected, max pooling and convolution layers.

The efficacy of the designed learning model architecture was compared against other available pre-trained CNN models such as ResNet50 and VGG16 to further verify the effectiveness of the selected CNN layered architecture. The classification of fMRI images was also compared using the SVM learning model designed with CNN architecture as it gives a better pattern recognition mechanism after CNN. Some of the existing studies have also used SVM for fMRI classification (Rubasinghe \& Meedeniya, 2020). The obtained results show that the designed CNN architecture has given the best classification accuracy for the considered fMRI dataset, compared to other pre-trained models and SVM as in Table 3.

\section{DISCUSSION}

\section{Main Contribution of the Study}

The key focused areas of this study are fMRI data processing, feature extraction and classification to have an accurate model for ADHD identification. The study was able to identify optimal data 

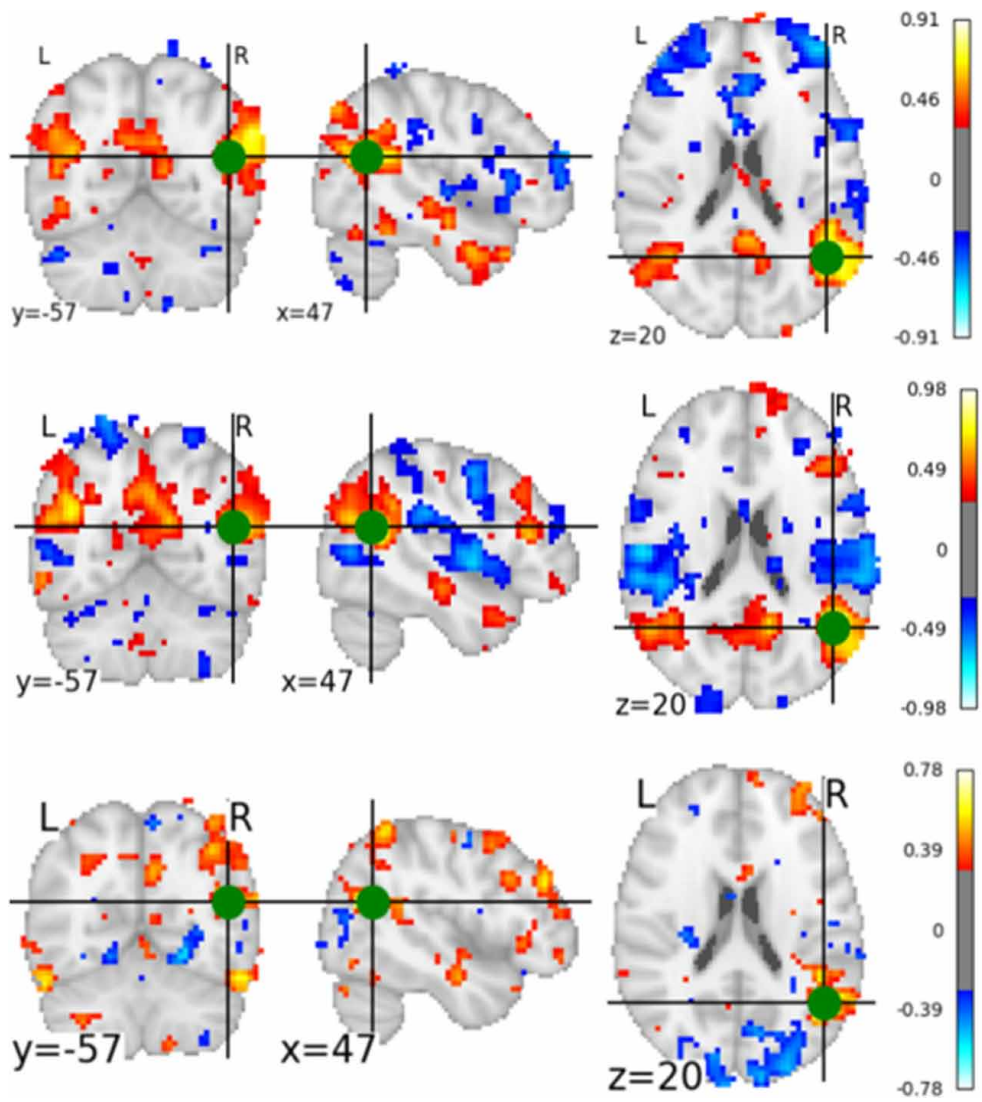

(a) Correlation between $\mathrm{ReHo}$ and SBC

(b) Correlation between

ReHo and fALFF

(c) Correlation between fALFF and SBC

Table 3. Comparison of results with different learning models

\begin{tabular}{|l|l|l|l|l|}
\hline Learning model & $\begin{array}{l}\text { Feature } \\
\text { any other } \\
\text { detail) }\end{array}$ & Accuracy & Specificity & Sensitivity \\
\hline ResNet50 (Residual Network) & SBC & $67.06 \%$ & $55.12 \%$ & $68.11 \%$ \\
\hline VGG16 (A CNN by Visual Geometry Group, Oxford) & SBC & $68.13 \%$ & $56.44 \%$ & $69.54 \%$ \\
\hline SVM (Support Vector Machine) & SBC & $67.51 \%$ & $52.11 \%$ & $66.48 \%$ \\
\hline CNN with SBC & SBC & $85.36 \%$ & $66.54 \%$ & $72.80 \%$ \\
\hline
\end{tabular}

processing techniques, while obtaining the prominent feature extraction techniques by experimenting with seed-based correlation, ReHo and fALFF. Furthermore, CNN model was used as the classification model, considering the DMN region after several evaluations for all the techniques in the considered process. CNN learning model along with the feature extraction method of seed-based correlation acquired an accuracy of $85.36 \%$ for pre-processed data by SPM12 and $83.4 \%$ for pre-processed data by C-PAC, as shown in Table 2 and Figure 7. 


\section{Result Comparison with Related Studies}

The proposed study focused on ADHD identification using fMRI data by extracting the most prominent features of fALFF, ReHo, and SBC to get the regional activities in the brain. DMN was used to study the abnormal activities while using that DMN region with SBC to extract features. The novelty of the study is more emphasized by pre-processing fMRI raw data and the optimal feature extraction methodology. Moreover, the CNN model that has been used as the learning model, was able to drive an accurate model with the pre-processed and feature extracted fMRI data. As an extension to our previous study on ADHD identification using eye movement data with a rule-based approach (De Silva et al., 2019b), and ADHD-Care_v1 using the pre-processed fMRI data with CNN technique (Ariyarathne, 2020), in this study, we have implemented the ADHD-Care_v2 which focuses mainly on un-processed fMRI data for the ADHD identification using CNN.

Table 4 states the comparison of the proposed study with the related work. The consideration of different feature types of fMRI data is used to identify the important aspects such as spatial distribution (Cortese et al., 2012), region-wise analysis, and the complementary and novel feature exposure. Cortical features specify the connectivity patterns of sensory areas in the human brain. The spatial and temporal features consist of spatial dependencies between voxels and the related time components of the functional MRI data, respectively. Connectivity features include the correlation measures between different brain regions. Frequency feature indicates the selection of the highest frequency of the voxel in the BOLD signal recorded as fMRI data after converting from the time domain to the frequency domain (Kuang et al., 2014b).

Among the features found in the related studies, cortical features representing the brain structure have been focused on both Peng et al. and Deshpande et al. Apart from that spatial features focusing on the spatial distribution of the brain areas, Mao et al. and Kuang et al. have been using deep belief networks and 4D CNN for different experimental setups. These studies have been used ELM and FCC (Fully Connected Cascade) ANN as the classification method and obtained high accuracy values, but have not been able to narrow down the areas of interest which are found to be highly active in ADHD subjects. Since the deep learning methods are not representable, having a specification of the area of interest is vital in medical imaging analysis.

As another approach, the brain neural connectivity has been studied by the neural activity in Sato et al. using fMRI data. They have used SVM classifier by considering different combinations of features such as ReHo, fALFF and RSN. Considering different combinations, they have shown a classification accuracy of $67 \%$. Seed based approach with CNN classification for main DMN regions that are activated in ADHD subjects was implemented as a prior version, ADHD-Care_v1 (Ariyarathne et al., 2020). In that study, an accuracy of $85.21 \%$ was obtained for the MPC region with a sensitivity of $72.80 \%$. Additionally, Rubasinghe \& Meedeniya, have shown similar classification accuracy values using different learning models; however, it is limited with customizable classifiers (Rubasinghe \& Meedeniya, 2020).

The proposed methodology using SBC in this paper has been able to address most of the issues in the previous work including the consideration of brain regions of interest other than the whole brain aspect, application of data augmentation to eliminate any imbalance in the dataset. Moreover, this study is based on an ensemble model consists of all DMN regions. An accuracy of $85.36 \%$ was obtained with the assignment of the appropriate weights for each DMN region model according to the accuracy values obtained for each DMN regional model. Thus, the solution was able to achieve high accuracy supported by above-average sensitivity and specificity values. Moreover, one of the main contributions of the proposed study is to show that the seed-based correlation is one of the optimal feature extraction technique for fMRI classification in ADHD identification process. The results show that the proposed methodology, based on seed-based correlations of main DMN areas, has high performance compared to the other features and prior works. Both pre-processing approaches for fMRI data with C-PAC pre-processing pipeline and SPM12 have obtained similar values with a slight difference in accuracy, specificity, and sensitivity. Also, the classification with pre-processed 
Table 4. Comparison of results with related studies

\begin{tabular}{|c|c|c|c|c|c|c|c|c|}
\hline \multirow{2}{*}{$\begin{array}{l}\text { Related work on ADHD } \\
\text { classification }\end{array}$} & \multirow{2}{*}{$\begin{array}{c}\text { Classification } \\
\text { Technique }\end{array}$} & \multicolumn{4}{|c|}{ Feature Type } & \multicolumn{3}{|c|}{ Results } \\
\hline & & $\begin{array}{l}\text { Cortical } \\
\text { features }\end{array}$ & $\begin{array}{c}\text { Spatio } \\
\text { temporal }\end{array}$ & \begin{tabular}{|c|} 
Connectivity \\
features
\end{tabular} & $\begin{array}{c}\text { Frequency } \\
\text { feature }\end{array}$ & $\begin{array}{c}\text { Accuracy } \\
(\%)\end{array}$ & $\begin{array}{c}\text { Specificity } \\
(\%)\end{array}$ & $\begin{array}{c}\text { Sensitivity } \\
(\%)\end{array}$ \\
\hline (Peng et al., 2013) & ELM & $\mathrm{X}$ & & & & 84.73 & & \\
\hline (Mao et al., 2019) & 4D CNN & & $\mathrm{X}$ & & & 71.30 & 73.20 & 69.70 \\
\hline (Kuang et al., 2014a) & DBN & & $\mathrm{x}$ & & & 72.73 & & \\
\hline $\begin{array}{l}\text { (Deshpande, Wang, } \\
\text { Rangaprakash \& } \\
\text { Wilamowski, 2015) }\end{array}$ & FCC ANN & $\mathrm{X}$ & & & & 90.0 & & \\
\hline (Sato et. al, 2012) & SVM & & & $\mathrm{X}$ & & 67.0 & 68.00 & 46.20 \\
\hline $\begin{array}{c}\text { (Rubasinghe \& } \\
\text { Meedeniya, 2020) }\end{array}$ & $\begin{array}{l}\text { SVM, NB, } \\
\text { KNN }\end{array}$ & & & $\mathrm{X}$ & & 86.00 & & \\
\hline \multirow[t]{4}{*}{ (Ariyarathne et al., 2020) } & \multirow[t]{4}{*}{$\mathrm{CNN}$} & & & PCC & & 84.84 & 65.22 & 71.12 \\
\hline & & & & LTJ & & 85.05 & 66.31 & 72.23 \\
\hline & & & & RTJ & & 84.62 & 64.12 & 70.85 \\
\hline & & & & MPC & & 85.21 & 66.41 & 72.80 \\
\hline $\begin{array}{c}\text { Proposed ADHD-Care_v2 } \\
\text { model }\end{array}$ & 3D CNN & & & $\mathrm{x}$ & & 85.36 & 66.54 & 72.80 \\
\hline
\end{tabular}

data has shown higher accuracy compared to the use of raw data without applying any pre-processing techniques.

Further, FCC neural network architectures are bound to be overfitting without a proper way of handling overfitting issues. The recommended practices include regularization and data augmentation (Cogswell et. al, 2015; Xu, et. al, 2019). Although the work presented by Deshpande et al., has used an FCC architecture, and obtained 90\% accuracy value, it has a higher potential of overfitting without the usage of the techniques such as regularization and data augmentation. In contrast, the proposed solution of this paper has used CNN with a final layer of fully connected layer, whereas Deshpande et. al. have used a cascading architecture of fully connected layers. However, using an array of fully connected layers in a neural network is bound to be overfitting (Xu, et. al, 2019). Further, to prevent the overfitting, in this proposed solution we have used L2 regularization and data augmentation. Because of that the overfitting of the presented model has been limited, (Jaiswal et. al, 2018; Takahashi et. al, 2016) and was able to achieve $85.36 \%$ accuracy.

\section{Future Research Directions}

As this study is mainly based on the DMN brain region, this can be further improved by exploring different brain regions with the un-processed fMRI data. Furthermore, ADHD subtype identification can also be done as a future work, which will be an extension to our study. Although only fMRI data is considered for the proposed ADHD identification, this research can be extended to a framework that can input other psychophysiological measures such as eye movement data, EEG, structural MRI. Moreover, such data sources can be experimented by combining with fMRI data, which will be a novel future work in the research field of ADHD identification. Thus, this approach can be extended to a generic framework (Meedeniya \& Rubasinghe, 2020), that supports the classification of different psychophysiological data related to neurological disorders. However, acquiring appropriate datasets with the correct labelling of disorder types and a large number of datasets are some of the challenges associated with health-care research due to disclosure of personal data. Further, the classification accuracy and performance can be improved by applying Deep Probabilistic Programming based approaches to process medical images (Rubasinghe \& Meedeniya, 2019). 


\section{CONCLUSION}

The advancement of medical image processing supports immensely to analyse clinical data. ADHD is a chronic psychiatric disorder resulting in behavioural issues that affect social interaction, communication and leads to many other comorbid disorders. Hence, early diagnosis of ADHD is important to reduce the risk of having severe symptoms. This paper has addressed fMRI image processing, feature extraction and classification approach that enables accurate ADHD subject classification. Mainly, this study is based on identifying the optimal data processing and feature extraction methods based on seed-based correlation, ReHo and fALFF for CNN based learning model that considers the entire DMN region coordinates. The classification accuracy for a seed-based approach using pre-processed by SPM12 and C-PAC has obtained $85.36 \%$ and $83.4 \%$ for selected DMN regions, respectively.

This work can be further extended by appraising different networks other than DMN such as a motor network to derive the seed-based correlations. The proposed solution can be further developed to identify the ADHD subtypes; inattentively, impulsivity and hyperactivity. In datasets point of view, a combination of different data sources such as EEG, eye movement data, structural MRI can be used together with fMRI data to acquire balanced sensitivity and specificity values. 


\section{REFERENCES}

Abreu, R., Leal, A., \& Figueiredo, P. (2018). EEG-informed fMRI: A review of data analysis methods. Frontiers in Human Neuroscience, 12, 29. doi:10.3389/fnhum.2018.00029 PMID:29467634

ADHD-care. (2019). Retrieved 10 October 2019, from http://blooming-sands-73478.herokuapp.com/

Aiello, M., Salvatore, E., Cachia, A., Pappata, S., Cavaliere, C., Prinster, A., Nicolai, E., Salvatore, M., Baron, J.-C., \& Quarantelli, M. (2015). Relationship between simultaneously acquired resting-state regional cerebral glucose metabolism and functional MRI: A PET/MRI hybrid scanner study. NeuroImage, 113, 111-121. doi:10.1016/j.neuroimage.2015.03.017 PMID:25791784

American Psychiatric Association. (2013). Diagnostic and statistical manual of mental disorders (DSM-5®). American Psychiatric Pub.

Ariyarathne, G., De Silva, S., Dayarathna, S., Meedeniya, D., \& Jayarathna, S. (2020). ADHD Identification using Convolutional Neural Network with Seed-based Approach for fMRI Data. 9th International Conference on Software and Computer Applications (ICSCA 2020), (pp. 31-35), ACM doi:10.1145/3384544.3384552

Ashburner, J., Barnes, G., Chen, C., Daunizeau, J., Flandin, G., Friston, K., \& Penny, W. (2014). SPM12 manual. Wellcome Trust Centre for Neuroimaging.

Bellec, P., Chu, C., Chouinard-Decorte, F., Benhajali, Y., Margulies, D. S., \& Craddock, R. C. (2017). The neuro bureau ADHD-200 pre-processed repository. NeuroImage, 144, 275-286. doi:10.1016/j.neuroimage.2016.06.034 PMID:27423255

Brihadiswaran, G., Haputhanthri, D., Gunathilaka, S., Meedeniya, D., \& Jayarathna, S. (2019). EEG-based processing and classification methodologies for Autism Spectrum Disorder: A Review. Journal of Computer Science, 15(8), 1161-1183.

Cogswell, M., Ahmed, F., Girshick, R., Zitnick, L., \& Batra, D. (2015). Reducing overfitting in deep networks by decorrelating representations. arXiv preprint arXiv: 1511.06068

Cortese, S., Kelly, C., Chabernaud, C., Proal, E., Di Martino, A., Milham, M. P., \& Castellanos, F. X. (2012). Toward systems neuroscience of ADHD: A meta-analysis of $55 \mathrm{fMRI}$ studies. The American Journal of Psychiatry, 169(10), 1038-1055. doi:10.1176/appi.ajp.2012.11101521 PMID:22983386

Craddock, C., Sikka, S., Cheung, B., Khanuja, R., Ghosh, S. S., Yan, C., \& Colcombe, S. (2013). Towards automated analysis of connectomes: The configurable pipeline for the analysis of connectomes (c-pac). Frontiers in Neuroinformatics, 42.

Dai, D., Wang, J., Hua, J., \& He, H. (2012). Classification of ADHD children through multimodal magnetic resonance imaging. Frontiers in Systems Neuroscience, 6, 63. doi:10.3389/fnsys.2012.00063 PMID:22969710

De Silva, S., Dayarathna, S., Ariyarathne, G., Meedeniya, D., \& Jayarathna, S. (2019a). A Survey of Attention Deficit Hyperactivity Disorder Identification Using Psychophysiological Data. International Journal of Online And Biomedical Engineering, 15(13), 61-76. doi:10.3991/ijoe.v15i13.10744

De Silva, S., Dayarathna, S., Ariyarathne, G., Meedeniya, D., Jayarathna, S., Michalek, A. M., \& Jayawardena, G. (2019b). A Rule-Based System for ADHD Identification using Eye Movement Data. In 2019 Moratuwa Engineering Research Conference (MERCon). IEEE (pp. 538-543), Sri Lanka. doi: 10.1109/MERCon.2019.8818865

Deshpande, G., Wang, P., Rangaprakash, D., \& Wilamowski, B. (2015). Fully connected cascade artificial neural network architecture for attention deficit hyperactivity disorder classification from functional magnetic resonance imaging data. IEEE Transactions on Cybernetics, 45(12), 2668-2679. doi:10.1109/TCYB.2014.2379621 PMID:25576588

Glover, G. H. (2011). Overview of functional magnetic resonance imaging. Neurosurgery Clinics, 22(2), 133-139. PMID:21435566

Hao, A. J., He, B. L., \& Yin, C. H. (2015). Discrimination of ADHD children based on Deep Bayesian Network. In 2015 IET International Conference on Biomedical Image and Signal Processing (ICBISP) (pp. 1- 6). IEEE. doi:10.1049/cp.2015.0764 
Haputhanthri, D., Brihadiswaran, G., Gunathilaka, S., Meedeniya, D., Jayarathna, S., Jaime, M., \& Jayawardena, Y. (2019). An EEG based Channel Optimized Classification Approach for Autism Spectrum Disorder. In Moratuwa Engineering Research Conference (MERCon), (pp. 123-128). IEEE. doi:10.1109/MERCon.2019.8818814

Hussain, Z., Gimenez, F., Yi, D., \& Rubin, D. (2017). Differential data augmentation techniques for medical imaging classification tasks. In AMIA Annual Symposium Proceedings (pp. 979). American Medical Informatics Association.

Image Preprocessing - Keras Documentation. (2019). Retrieved 10 October 2019, from https://keras.io/ preprocessing/image/

Jaiswal, S., Mehta, A., \& Nandi, G. C. (2018, June). Investigation on the Effect of L1 an L2 Regularization on Image Features Extracted Using Restricted Boltzmann Machine. In 2nd International Conference on Intelligent Computing and Control Systems (ICICCS) (pp. 1548-1553). IEEE. doi:10.1109/ICCONS.2018.8663071

Kaur, S., Arun, P., Singh, S., \& Kaur, D. (2018, December). EEG Based Decision Support System to Diagnose Adults with ADHD. In 2018 IEEE Applied Signal Processing Conference (ASPCON) (pp. 87-91). IEEE. doi:10.1109/ASPCON.2018.8748412

Kuang, D., Guo, X., An, X., Zhao, Y., \& He, L. (2014a). Discrimination of ADHD based on fMRI data with deep belief network. In International Conference on Intelligent Computing (pp. 225-232). Springer. doi:10.1007/9783-319-09330-7_27

Kuang, D., \& He, L. (2014b). Classification on ADHD with Deep Learning. In International Conference on Cloud Computing and Big Data (pp. 27-32), IEEE.

Lenartowicz, A., \& Loo, S. K. (2014). Use of EEG to diagnose ADHD. Current Psychiatry Reports, 16(11), 498. doi:10.1007/s11920-014-0498-0 PMID:25234074

Mao, Z., Su, Y., Xu, G., Wang, X., Huang, Y., Yue, W., \& Xiong, N. (2019). Spatio-temporal deep learning method for ADHD fMRI classification. Information Sciences, 499, 1-11. doi:10.1016/j.ins.2019.05.043

Meedeniya, D. A., \& Rubasinghe, I. D. (2020). A Review of Supportive Computational Approaches for Neurological Disorder Identification. In T. Wadhera \& D. Kakkar (Eds.), Interdisciplinary Approaches to Altering Neurodevelopmental Disorders. Chapter 16, pp. 271-302, IGI Global. doi: 10.4018/978-1-7998-3069-6.ch016

Meraj, S. S., Yaakob, R., Azman, A., Rum, S. N. M., \& Nazri, A. S. A. (2019). Artificial Intelligence in Diagnosing Tuberculosis: A Review. International Journal on Advanced Science. Engineering and Information Technology, 9(1), 81-91.

Metin, B., Krebs, R. M., Wiersema, J. R., Verguts, T., Gasthuys, R., van der Meere, J., \& Sonuga-Barke, E. (2015). Dysfunctional modulation of default mode network activity in attention-deficit/hyperactivity disorder. Journal of Abnormal Psychology, 124(1), 208-214. doi:10.1037/abn0000013 PMID:25314265

Miao, B., \& Zhang, Y. (2017, July). A feature selection method for classification of ADHD. In 2017 4th International Conference on Information, Cybernetics and Computational Social Systems (ICCSS) (pp. 21-25). IEEE. doi:10.1109/ICCSS.2017.8091376

Omidvarnia, A., Pedersen, M., Walz, J. M., Vaughan, D. N., Abbott, D. F., \& Jackson, G. D. (2016). Dynamic regional phase synchrony (DRePS): An Instantaneous Measure of Local fMRI Connectivity within Spatially Clustered Brain Areas. Human Brain Mapping, 37(5), 1970-1985. doi:10.1002/hbm.23151 PMID:27019380

Parida, S., Dehuri, S., \& Cho, S. B. (2015). Machine Learning Approaches for Cognitive State Classification and Brain Activity Prediction: A Survey. Current Bioinformatics, 10(4), 344-359. doi:10.2174/15748936096 66140820224846

Penberthy, J. K., Cox, D., Breton, M., Robeva, R., Kalbfleisch, M. L., Loboschefski, T., \& Kovatchev, B. (2005). Calibration of ADHD assessments across studies: A meta-analysis tool. Applied Psychophysiology and Biofeedback, 30(1), 31-51. doi:10.1007/s10484-005-2172-0 PMID:15889584

Peng, X., Lin, P., Zhang, T., \& Wang, J. (2013). Extreme learning machine-based classification of ADHD using brain structural MRI data. PLoS One, 8(11), e79476. doi:10.1371/journal.pone.0079476 PMID:24260229 
Polanczyk, G., De Lima, M. S., Horta, B. L., Biederman, J., \& Rohde, L. A. (2007). The worldwide prevalence of ADHD: A systematic review and metaregression analysis. The American Journal of Psychiatry, 164(6), 942-948. doi:10.1176/ajp.2007.164.6.942 PMID:17541055

Rubasinghe, I. D., \& Meedeniya, D. A. (2019). Ultrasound Nerve Segmentation Using Deep Probabilistic Programming, Journal of ICT Research and Applications, 13(3), 241-256, ITB Journal Publisher. doi: 10.5614\%2Fitbj.ict.res.appl.2019.13.3.5

Rubasinghe, I. D., \& Meedeniya, D. A. (2020). Automated Neuroscience Decision Support Framework. In Deep Learning Techniques for Biomedical and Health Informatics. Academic Press. doi:10.1016/B978-0-12819061-6.00013-6

Ruder, S. (2016). An overview of gradient descent optimization algorithms. arXiv preprint arXiv:1609.04747

Sato, J. R., Hoexter, M. Q., Fujita, A., \& Rohde, L. A. (2012). Evaluation of pattern recognition and feature extraction methods in ADHD prediction. Frontiers in Systems Neuroscience, 6, 68. doi:10.3389/fnsys.2012.00068 PMID:23015782

Siuly, S., \& Zhang, Y. (2016). Medical big data: Neurological diseases diagnosis through medical data analysis. Data Science and Engineering, 1(2), 54-64. doi:10.1007/s41019-016-0011-3

Song, X. W., Dong, Z. Y., Long, X. Y., Li, S. F., Zuo, X. N., Zhu, C. Z., \& Zang, Y. F. (2011). REST: A toolkit for resting-state functional magnetic resonance imaging data processing. PLoS One, 6(9), e25031. doi:10.1371/ journal.pone.0025031 PMID:21949842

Takahashi, N., Gygli, M., Pfister, B., \& Van Gool, L. (2016). Deep convolutional neural networks and data augmentation for acoustic event detection. arXiv preprint, arXiv:1604.07160

Uddin, L. Q., Kelly, A. C., Biswal, B. B., Margulies, D. S., Shehzad, Z., Shaw, D., Ghaffari, M., Rotrosen, J., Adler, L. A., Castellanos, F. X., \& Milham, M. P. (2008). Network homogeneity reveals decreased integrity of default-mode network in ADHD. Journal of Neuroscience Methods, 169(1), 249-254. doi:10.1016/j. jneumeth.2007.11.031 PMID:18190970

Wen, D., Wei, Z., Zhou, Y., Li, G., Zhang, X., \& Han, W. (2018). Deep learning methods to process fMRI data and their application in the diagnosis of cognitive impairment: A brief overview and our opinion. Frontiers in Neuroinformatics, 12, 23. doi:10.3389/fninf.2018.00023 PMID:29755334

Xiao, C., Ye, J., Esteves, R. M., \& Rong, C. (2016). Using Spearman's correlation coefficients for exploratory data analysis on big dataset. Concurrency and Computation, 28(14), 3866-3878. doi:10.1002/cpe.3745

Xu, Q., Zhang, M., Gu, Z., \& Pan, G. (2019). Overfitting remedy by sparsifying regularization on fully-connected layers of CNNs. Neurocomputing, 328, 69-74. doi:10.1016/j.neucom.2018.03.080

Zang, Y., Jiang, T., Lu, Y., He, Y., \& Tian, L. (2004). Regional homogeneity approach to fMRI data analysis. Neurolmage, 22(1), 394-400. doi:10.1016/j.neuroimage.2003.12.030 PMID:15110032

Zou, L., Zheng, J., Miao, C., Mckeown, M., \& Wang, Z. (2017). 3D CNN Based Automatic Diagnosis of Attention Deficit Hyperactivity Disorder Using Functional and Structural MRI. IEEE Access : Practical Innovations, Open Solutions, 5, 23626-23636. doi:10.1109/ACCESS.2017.2762703

Zou, Q. H., Zhu, C. Z., Yang, Y., Zuo, X. N., Long, X. Y., Cao, Q. J., \& Zang, Y. F. (2008). An improved approach to detection of amplitude of low-frequency fluctuation (ALFF) for resting-state fMRI: Fractional ALFF. Journal of Neuroscience Methods, 172(1), 137-141. doi:10.1016/j.jneumeth.2008.04.012 PMID:18501969 
S. De Silva is an undergraduate student at the Department of Computer Science and Engineering, University of Moratuwa, Sri Lanka. She has research experience in Biomedical, Machine learning and Data Mining. Her Orcid Id is https://orcid.org/0000-0002-4526-9586

S. Dayarathna is an undergraduate student at the Department of Computer Science and Engineering, University of Moratuwa, Sri Lanka. She has research experience in Biomedical, Machine Learning and Data Mining. Her Orcid Id is https://orcid.org/0000-0001-8669-2893

G. Ariyarathne is an undergraduate student at the Department of Computer Science and Engineering, University of Moratuwa, Sri Lanka. She has research experience in Machine Learning, Data Mining, Biomedical and Computer Security. Her Orcid Id is https://orcid.org/0000-0003-4205-6574

D. Meedeniya $(P h D)$ is a Senior Lecturer in the Department of Computer Science and Engineering, at the University of Moratuwa, Sri Lanka. She holds a PhD in Computer Science from the University of St Andrews, United Kingdom. Her main research interests are Software modelling and design, Workflow tool support for bioinformatics, Data Visualization and Recommender systems. She is a Fellow of HEA(UK), MIET, MIEEE and a Charted Engineer registered at EC (UK). https://orcid.org/0000-0002-4520-3819

Sampath Jayarathna $(P h D)$ is an Assistant Professor of Computer Science at Old Dominion University, where he is associated with Web Science and Digital Libraries (WS-DL) research group. Dr. Jayarathna earned a PhD in Computer science from the Texas A\&M University College Station in 2016. His research interest includes machine learning, information retrieval, data science, eye tracking, and brain computer interfacing. Dr. Jayarathna is a member of ACM, IEEE, and Sigma XI. 